\title{
Atualização no Tratamento da Diverticulite Aguda do Cólon
}

\author{
Recent Evidences in the Management of Acute Diverticulitis
}

\author{
ANDRÉ RONCON DIAS ${ }^{1}$;ANACECÍLIANEIVAGONDIM²; SÉRGIO CARLOS NAHAS $^{3}$ \\ ${ }^{1}$ Médico preceptor da Disciplina de Cirurgia do Aparelho Digestivo do HCFMUSP; ${ }^{2}$ Médica residente de cirurgia do \\ HCFMUSP; ${ }^{3}$ Professor livre docente da Faculdade de Medicina da Universidade de São Paulo. Diretor do Serviço de \\ Cirurgia do Cólon e Reto do HCFMUSP.
}

DIAS AR; GONDIM ACN; NAHAS SC. Atualização no Tratamento da Diverticulite Aguda do Cólon. Rev bras Coloproct, 2009;29(3): 363-371.

RESUMO: Nas últimas décadas a incidência da moléstia diverticular do cólon e de suas complicações tem aumentado. Durante esse período o número de publicações a respeito do tema se expandiu, bem como as opções terapêuticas. Apesar disso, muitas dúvidas persistem e as decisões terapêuticas continuam relativamente imutadas, baseadas muitas vezes em dados antigos e de baixo poder estatístico. Nesta revisão apresentamos as evidências científicas atuais acerca da terapêutica desta complexa patologia.

Descritores: Moléstia diverticular do cólon, diverticulite aguda, tratamento.

\section{INTRODUÇÃO}

A moléstia diverticular do cólon é uma patologia decorrente dos hábitos alimentares da vida moderna. Associada a uma dieta pobre em fibras sua incidência tem crescido nas últimas décadas. Dados norte-americanos demonstram que em 1998 ocorreram 120.500 internações nos E.U.A. devido a doença diverticular, enquanto em 2005 ocorreram 151.900 internações (acréscimo de $26 \%$ em 7 anos). No mesmo período o número de intervenções cirúrgicas subiu $29 \%$ (de 16.100 para 22.500). ${ }^{1}$

A doença diverticular pode ocorrer em todo o cólon, porém a região mais frequentemente acometida é o sigmoide e raramente há doença abaixo da reflexão peritoneal. A doença diverticular do cólon direito, padrão predominante nas populações asiáticas, raramente acomete a população ocidental.

Estima-se que $30 \%$ da população com mais de 60 anos e $60 \%$ dos indivíduos com mais de 80 anos sejam afetados. ${ }^{2}$ Com o envelhecimento global da po- pulação, espera-se que a doença diverticular e suas complicações representem uma porcentagem cada vez maior do atendimento do cirurgião, tanto em consultórios como em emergências. ${ }^{3}$

O foco desta revisão, dentre a miríade de manifestações clínicas possíveis da doença diverticular (ausência de sintomas, queixas vagas e inespecíficas, sangramento intestinal etc.) será o abdome agudo (diverticulite) e os dados mais recentes acerca do tratamento desta que é a manifestação mais grave e mórbida da diverticulose cólica.

Estima-se que 10-25\% dos pacientes com diverticulose cólica evoluirão com diverticulite, sendo que $95 \%$ das vezes essa complicação ocorrerá no sigmoide. ${ }^{4,5}$ Destes, um quarto evoluirá com complicações potencialmente ameaçadoras à vida como: perfuração, fístula, obstrução ou estenose. ${ }^{3}$

Ainda hoje e apesar de existirem guidelines de diversas sociedades acerca do tratamento da diverticulite aguda, muitas dúvidas persistem: Quando indicar a cirurgia? Qual cirurgia devemos realizar? Qual

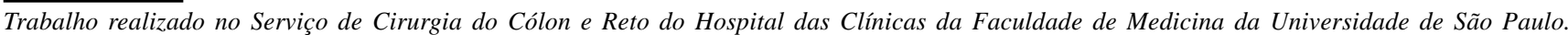

Recebido em 30/06/2009

Aceito para publicação em 27/07/2009 
o melhor momento para operar o paciente? Qual deve ser a via de acesso? E quais são as evidências científicas que sustentam as decisões tomadas?

Para responder a essas perguntas nos basearemos no grau de confiabilidade dos dados da literatura (Tabela 1).

\section{CONFIRMAÇÃO DIAGNÓSTICA}

A tomografia computadorizada (TC) é o melhor exame diagnóstico (grau de recomendação A). ${ }^{6}$ Seu valor preditivo positivo é de $88 \%$ na inflamação pericólica e de $100 \%$ quando há espessamento da parede cólica maior que $10 \mathrm{~mm} .{ }^{7}$ Fator de suma importância é a severidade da diverticulite observada na TC que se correlaciona com o risco de falha do tratamento clínico e necessidade de cirurgia. ${ }^{8}$

A TC de abdome e pelve também permite classificar a diverticulite aguda em não complicada e complicada (abscesso, fístula, obstrução, perfuração livre) norteando, junto à classificação de Hinchey, o tratamento a ser instituído (Tabela 2).

\section{TRATAMENTO}

\section{Diverticulite aguda não complicada}

O tratamento da diverticulite aguda não complicada é clínico e pode ser ambulatorial com dieta rica em fibras e antibioticoterapia oral de amplo espectro (cobertura para gram negativos e anaeróbios). Pacientes cujo tratamento ambulatorial falha ou está impossibilitado (ex: pacientes idosos ou reticentes, imunossuprimidos, etc) devem ser internados. 9, 10
O sucesso do tratamento clínico nestes casos é de $85 \%$. Destes uma fração significativa terá recorrência da diverticulite, geralmente dentro de 1 ano. ${ }^{8}$ A suplementação oral de fibras dietéticas reduz o risco de recorrência. ${ }^{5}$

Diverticulite aguda complicada - Hinchey II

Abscessos pequenos $(<2 \mathrm{~cm})$ e/ou mesocólicos podem ser tratados clinicamente. Abscessos maiores devem ser tratados com drenagem percutânea guiada por ultrassonografia (USG) ou TC, associados a antibioticoterapia (grau de recomendação B), mantendo-se o doente internado. ${ }^{6}$

A drenagem percutânea permite que cirurgias de urgência/emergência sejam evitadas, reduzindo o número de cirurgias em múltiplas etapas e de estomias. ${ }^{8}$

\section{III/IV}

Diverticulite aguda complicada - Hinchey

A cirurgia de urgência está indicada na presença de peritonite. Pacientes com abscessos não passíveis de drenagem percutânea ou cujo tratamento clínico falhou também devem ser operados (grau de recomendação B).

Tabela 2 - Classificação de Hinchey.

\begin{tabular}{ll}
\hline I & Abscesso pericólico ou flegmão \\
II & Abscesso intra-abdominal ou retroperitoneal \\
III & Peritonite purulenta \\
IV & Peritonite fecal \\
\hline
\end{tabular}

\section{Tabela 1}

\section{Fonte da evidência}

I Meta-análises de estudos bem desenhados/ estudos randomizados de alto poder estatístico

II Estudos experimentais bem desenhados/ estudos randomizados de menor poder estatístico

III Estudos não-randomizados, cohortes, comparativos

IV Estudos comparativos, estudos de casos

V Relatos de casos e exemplos clínicos

\section{Grau de recomendação}

A Evidência tipo I ou múltiplos estudos II, III ou IV

B Evidências tipo II, III ou IV

C Evidências tipo II, III ou IV com achados inconsistentes

D Pouca ou nenhuma evidência sistemática 
As prioridades na cirurgia de urgência são: controlar a sepse, ressecar o tecido doente e restabelecer o trânsito intestinal, nessa ordem de importância. Dentre as várias opções cirúrgicas podemos citar a cirurgia em três tempos (com colostomia e drenagem na primeira etapa), a cirurgia de Hartmann, a ressecção com anastomose primária com ou sem estomia de proteção e, mais recentemente, a limpeza e drenagem da cavidade pela via laparoscópica com re-abordagem eletiva.

Quanto à cirurgia em três etapas, esta é raramente indicada, pois se associa a uma maior morbimortalidade (mortalidade de $26 \%$ vs $7 \%$ quando realiza-se a sigmoidectomia na primeira abordagem). ${ }^{12}$ Assim, a ressecção do sigmoide deve, sempre que possível, ser realizada no primeiro tempo cirúrgico. A decisão de realizar ou não a anastomose repousa na experiência de cada cirurgião, que muitas vezes se norteia pelo grau de inflamação e contaminação local, condição clínica e comorbidades do paciente.

A cirurgia de Hartmann (sigmoidectomia com sepultamento do reto e colostomia terminal) continua sendo o procedimento mais realizado na urgência, mesmo sabendo-se da dificuldade de reconstrução do trânsito nos pacientes submetidos a essa operação (em até $1 / 3$ dos casos a colostomia se torna definitiva). ${ }^{1,13}$

Quando se realiza a anastomose primária a opção por uma estomia de proteção repousa nos achados intra-operatórios, estado global do paciente e experiência do cirurgião. A ileostomia em alça tem sido preferida pela maioria dos grupos, associando-se a menor morbi-mortalidade. $\mathrm{O}$ fato do cólon não estar preparado durante a cirurgia de urgência é outro entrave à realização da anastomose primária. Nestes casos, quando o cirurgião está habituado ao método, a lavagem intra-operatória é uma opção.

Uma nova alternativa cirúrgica recentemente relatada consiste na inspeção da cavidade, lavagem e colocação de dreno pela via laparoscópica (LAP), associada a antibioticoterapia, retirando o paciente da fase aguda crítica e permitindo realizar uma sigmoidectomia laparoscópica eletiva posteriormente. ${ }^{14}$ Esta abordagem é possível uma vez a peritonite secundária à diverticulite aguda é raramente consequência de uma grande perfuração do cólon, com necessidade de sutura (contrariamente a outras causas de peritonite, como as úlceras pépticas perfuradas). ${ }^{14} \mathrm{Um}$ grupo norteamericano relatou os resultados de 40 pacientes tratados dessa maneira com ótimos resultados num segui- mento médio de 96 meses. Entre os benefícios dessa abordagem estariam a menor agressão ao doente já debilitado, a possibilidade de evitar uma colostomia, um menor custo global do tratamento e, principalmente, a menor incidência e gravidade de complicações de ferida operatória. ${ }^{15}$

Bretagnol et al ${ }^{14}$ realizaram lavagem e drenagem laparoscópica em 24 pacientes com diverticulite perfurada ( 5 paciente com Hinchey II, 18 grau III e 1 grau IV). Os autores relatam $8 \%$ de morbidade, $16 \%$ de conversão, nenhum óbito e nenhuma re-operação. Todos os pacientes foram submetidos à sigmoidectomia laparoscópica eletiva, em média 3,5 meses após o episódio agudo. Segundo esta série a abordagem minimamente invasiva é uma alternativa promissora em pacientes selecionados, evitando a necessidade de estomia.

Outro estudo submeteu 100 pacientes com diverticulite aguda e perfuração (comprovada por radiografia ou TC) à laparoscopia. Todos foram estagiados por essa via conforme a classificação de Hinchey. Em 8 pacientes com peritonite fecal houve conversão para via aberta, sendo realizado o procedimento de Hartmann e em todos os demais (25 com diverticulite grau II e 67 com grau III) procedeu-se à lavagem da cavidade e drenagem pela via laparoscópica. Houve 4\% de morbidade e 3\% de mortalidade. Não houve infecção de ferida operatória. Dois pacientes apresentaram abscesso pélvico, um com boa resposta ao tratamento conservador (antibioticoterapia e punção guiada por TC) e outro com necessidade de re-abordagem (realizada cirurgia de Hartmann). Dois pacientes apresentaram diverticulite aguda não complicada, tratada com antibioticoterapia, e nenhum necessitou de re-intervenção cirúrgica por complicação de doença diverticular em seguimento médio de 36 meses. Os autores concluem que a lavagem laparoscópica é uma alternativa aceitável no tratamento da diverticulite graus II e III de Hinchey e questionam a obrigatoriedade da ressecção eletiva subsequente, como classicamente preconizado. ${ }^{16}$

Contudo, a lavagem laparoscópica não é adequada para todas as situações. Pacientes com peritonite fecal apresentam resultados ruins com o método, a maioria necessitando de re-operação precoce com sigmoidectomia. A ressecção também deve ser considerada nos pacientes que apresentam evolução arrastada após a lavagem. ${ }^{17}$ 


\section{Acesso cirúrgico na urgência}

A ressecção laparoscópica na diverticulite aguda é factível e segura. ${ }^{18,19,20}$

Conforme as equipes adquirem maior experiência os resultados são melhores e as contra-indicações menores. Um estudo multicêntrico prospectivo com 1545 pacientes separou os centros participantes em três grupos de acordo com o volume operatório em ressecções por doença diverticular (grupo I > 100 casos; grupo II 30-100 casos; grupo III < 30 casos). Foi observado que equipes com maior casuística indicam mais frequentemente a LAP, inclusive nos casos de diverticulite complicada (estes consistiam em $21 \%$ dos casos do grupo I, $9 \%$ no II e $8 \%$ no III). Mesmo com indicação mais abrangente, os grupos de maior experiência obtiveram taxas de morbidade comparáveis à dos grupos de menor volume, que indicam a LAP em casos mais favoráveis (a morbidade nos 3 grupos foi de $16 \%, 17 \%$ e $18 \%$, respectivamente). Também não houve diferença estatística nas taxas de mortalidade entre os três grupos. ${ }^{20}$

Uma análise com 55.949 pacientes demonstrou também que cirurgiões de maior volume apresentam uma chance 8,8 vezes maior de realizar a cirurgia pela via laparoscópica do que cirurgiões com baixo volume de casos. Pacientes operados em centros de maior volume também tiveram uma chance maior (três vezes) de serem submetidos à LAP. ${ }^{21}$

Comparando o desfecho de pacientes com diverticulite aguda complicada $(n=46)$ e não complicada $(\mathrm{n}=79)$ submetidos à ressecção laparoscópica, um estudo observou uma taxa global de conversão de $26 \%$, não havendo diferença estatística entre os dois grupos. A presença de cirurgia abdominal prévia foi o único fator estatisticamente significativo associado a uma maior taxa de conversão ( 37 vs $14 \%$ ). ${ }^{22}$

\section{Cirurgia eletiva}

A cirurgia deve ser realizada após a recuperação do paciente e sua indicação é baseada individualmente em cada caso (grau de recomendação B).

Atualmente podemos aceitar como indicação:

1. Após $1^{\circ}$ episódio de diverticulite complicada

2. Após drenagem percutânea de diverticulite complicada

3. Incapacidade de excluir doença neoplásica

A cirurgia após a drenagem percutânea visa evitar sepse recorrente, que ocorre em até $41 \%$ dos pacientes ${ }^{11}$ (grau de recomendação B).
Ao indicar a sigmoidectomia após um episódio de diverticulite não complicada tratada clinicamente, devemos levar em consideração a idade do paciente, suas comorbidades e se há persistência de sintomas (grau de recomendação B). ${ }^{23,24,25}$

A cirurgia após diverticulite não complicada é tema controverso. Em 2000 as sociedades Européia de Cirurgia Endoscópica, Americana de Cirurgiões Colorretais e Americana de Gastroenterologia divulgaram seus guidelines, todos recomendando o tratamento cirúrgico após duas crises de diverticulite aguda. Essa indicação repousava na revisão de Parks de 1969 com 521 pacientes que observou uma menor eficácia do tratamento conservador a cada novo episódio de diverticulite ( $70 \%$ sucesso no primeiro episódio vs $6 \%$ no terceiro). $5,23,26$

Porém, dados mais recentes apontam para uma direção diferente. Uma revisão sistemática da literatura incluindo 21 estudos (selecionados entre 1.360) avaliou o desfecho de pacientes com diverticulite aguda em que ambos tratamentos (conservador e cirúrgico) eram opções possíveis. O tratamento clínico foi realizado mais frequentemente ( 24.862 vs 6.504$)$ e os pacientes tratados clinicamente foram readmitidos por diverticulite aguda com maior frequência $(18,6 \%$ vs $6,1 \%)$. Pacientes com 1 ou 2 episódios de diverticulite aguda, tratados clinicamente, necessitaram de cirurgia por doença recorrente em um máximo de $45 \%$ dos casos, sendo que os estudos maiores mostraram taxas menores, de até $11 \% .{ }^{27}$

Um estudo multicêntrico retrospectivo incluindo 25.058 pacientes, com seguimento médio de 5,5 anos, notou que $19,6 \%$ dos doentes foram submetidos a tratamento cirúrgico na primeira internação. Dos 20.136 pacientes submetidos inicialmente a tratamento clínico, $19 \%$ foram readmitidos por diverticulite aguda. O risco de cirurgia de urgência aumentou 2,2 vezes a cada readmissão e os pacientes mais jovens tiveram um risco significativamente maior de readmissão e de cirurgia de urgência. Baseados nesses achados os autores recomendam individualizar a indicação cirúrgica. ${ }^{28}$

Em outro estudo multicêntrico retrospectivo, 2.366 pacientes (dentre 3.165 hospitalizados por diverticulite aguda) foram tratados clinicamente e acompanhados por 8,9 anos (média). Durante esse período a incidência de recorrência foi de $9,4 \%$ e apenas $3,9 \%$ dos pacientes foram readmitidos mais de uma vez. ${ }^{29}$

A baixa taxa de recorrência encontrada nesses estudos mais recentes argumenta contra a indica- 
ção de sigmoidectomia eletiva de rotina após o sucesso do tratamento clínico de pacientes com diverticulite aguda. Também não está claro se a morbi-mortalidade da cirurgia eletiva é inferior à da recorrência.

$\mathrm{O}$ tradicional argumento de que a cirurgia eletiva evitaria uma crise aguda complicada também não é válido, uma vez que o episódio agudo grave é na maioria das vezes a primeira manifestação da doença e $89,5 \%$ dos óbitos em doentes com crise aguda ocorrem em pacientes sem história prévia de diverticulite. ${ }^{30}$

Adicionalmente, um estudo prospectivo com 163 pacientes seguidos por 5 anos observou que a evolução da doença diverticular não complicada, diferentemente da forma complicada, é benigna, com uma incidência de complicações muito baixa. ${ }^{31}$

Ambrosetti analisou 118 pacientes com seguimento médio de 9,5 anos e notou que apenas a presença de idade jovem e diverticulite severa na TC foram fatores estatisticamente significativos de má evolução, porém a idade deixou de ser significativa após estratificação para severidade da doença. ${ }^{32}$

Numa coorte hipotética, visando determinar a melhor estratégia de tratamento após um episódio de diverticulite aguda tratada conservadoramente, foram simulados os desfechos clínicos e econômicos de pacientes de 35 e 50 anos. O tratamento cirúrgico após o $4^{\circ}$ episódio de diverticulite, em vez do segundo, resultou em uma menor incidência de óbitos, de colostomias, além de uma economia substancial (Tabela 3). ${ }^{33}$

Um estudo similar observou que a cirurgia após o terceiro episódio de diverticulite seria mais custo-efetiva do que após o primeiro ou segundo episódios. ${ }^{34}$

Portanto, não existe evidência de que devemos operar pacientes após um ou dois episódios de diverticulite aguda tratada conservadoramente com sucesso. Portanto, a indicação deve ser "sob medida",

Tabela 3 - Redução na incidência de óbitos $e$ estomias e economia (em dólares) ao indicar a cirurgia após o $4^{\circ}$ episódio de diverticulite, em vez do segundo. ${ }^{25}$

\begin{tabular}{lcc}
\hline & $<\mathbf{5 0}$ anos & $>\mathbf{5 0}$ anos \\
\hline Óbitos & $0,1 \%$ & $0,5 \%$ \\
Estomias & $2 \%$ & $0,7 \%$ \\
Economia & 5.429 & 1.035 \\
\hline
\end{tabular}

baseada no paciente, na sua idade, comorbidades e sintomatologia.

A indicação da cirurgia em pacientes jovens também é tema controverso, existindo muitos dados contraditórios na literatura. Alguns autores relatam uma maior agressividade quando a doença ocorre em pacientes mais jovens, havendo recorrência e complicações com maior frequência. ${ }^{28,35} \mathrm{Em}$ contrapartida, outros estudos não obtiveram os mesmos achados, além de, como vimos anteriormente, demonstrarem que ao estratificar a doença por gravidade, o risco independe da idade. ${ }^{31,36}$

Apesar de não haver consenso, o risco cumulativo de novas crises sempre será maior nos pacientes mais jovens, uma vez que a expectativa de vida é mais longa. Logo, é aconselhável informar ao paciente os riscos e benefícios, permitindo que a decisão seja tomada conjuntamente.

\section{Acesso cirúrgico eletivo}

A laparoscopia pode ser utilizada após diverticulite aguda tratada clinicamente, com segurança, eficácia e custos comparáveis ao acesso convencional, sendo apropriada em pacientes selecionados (grau de recomendação A).

Uma única instituição analisou retrospectivamente 500 pacientes operados pela via laparoscópica. As indicações mais frequentes foram: diverticulite recorrente (77\%), perfuração (10\%) e presença de fístulas (9\%). O tempo operatório médio foi de 120 minutos e a média de hospitalização foi quatro dias ( $p$ não significativo para doença com ou sem complicação). A incidência de morbidades maiores foi de $11 \%$, com um óbito registrado. A taxa de conversão foi de $2,1 \%$ nos casos de cirurgia eletiva, sendo em sua maioria devido a aderências. A taxa de conversão apresentou redução significativa (8\% para $1,5 \%)$ comparando os primeiros 100 casos com os 400 posteriores. ${ }^{37}$

Comparando os resultados da colectomia laparoscópica em 363 pacientes com doença diverticular e 313 com doença não diverticular, Schwandner et al ${ }^{38}$ concluíram que as taxas de conversão, complicação, mortalidade e necessidade de re-operação são similares entre os dois grupos.

Quando comparamos laparoscopia com a cirurgia aberta na doença diverticular há um único estudo multicêntrico, randomizado, o Sigma trial, com 52 pacientes em cada braço do estudo, pareados por gê- 
nero, idade, IMC, escore A.S.A., comorbidades e cirurgia abdominal prévia. Seus resultados em curto prazo mostraram que o grupo LAP teve maior tempo operatório, menor perda sanguínea e menor incidência de complicações maiores $(9,6 \%$ vs $25 \%)$. A taxa de conversão foi de $19,2 \%$ e a mortalidade de $1 \%$. Os pacientes do grupo LAP ainda apresentaram menor necessidade de analgesia sistêmica e menor tempo de internação. Os autores destacam que nesta casuística o acesso laparoscópico reduziu em 15,4\% as complicações maiores (deiscência de anastomose, abscesso intra-abdominal, sangramento pós-operatório com necessidade de hemotransfusão, reoperação em 30 dias). ${ }^{39}$

Shapiro et al ${ }^{40}$ comparando sigmoidectomias eletivas em pacientes com doença diverticular, sendo 166 abertas e 80 laparoscópicas, notaram que a LAP se associou a alta mais precoce ( 4 vs 8 dias), menor sangramento intra-operatório (100 vs $200 \mathrm{ml})$ e maior tempo cirúrgico (185 vs 153 minutos). As taxas de readmissão e recorrência foram similares. Houve conversão de $12,5 \%$ dos casos laparoscópicos.

Outro estudo comparativo, este com 95 colectomias laparoscópicas e 80 abertas, todas eletivas para doença diverticular, também observou que a LAP resulta significativamente em menor sangramento intraoperatório, menos complicações pós-operatórias, menor tempo de internação e menor íleo pós-operatório. Não houve diferença quanto ao tempo cirúrgico e complicações intra-operatórias. ${ }^{41}$

Senagore et al ${ }^{42}$ relataram que o tempo operatório e a taxa de readmissão foram similares entre os acessos em 132 sigmoidectomias eletivas por doença diverticular (61 laparoscopias e 71 abertas). O grupo LAP apresentou menor tempo de internação $(3,1 v s$ 6,8 dias), menor incidência de complicações pulmonares e de ferida operatória (1,6 vs 5,6\% e zero vs 7\%, respectivamente), com $6,6 \%$ de taxa de conversão. Houve um óbito no grupo laparoscópico. Os custos totais na LAP foram menores (3.458 vs 4.321 dólares).

A presença de fístulas após diverticulite aguda complicada tratada clinicamente, não deve ser uma contra-indicação ao acesso laparoscópico para uma equipe treinada em cirurgia videolaparoscópica. ${ }^{18,19}$ Nestes casos a curva de aprendizado parece influenciar significativamente o índice de conversões. Um grupo britânico divulgou seus resultados com 31 pacientes (22 com fístula colovesical e nove com fístula colovaginal) submetidos à sigmoidectomia LAP em um período de 11 anos. Os autores demonstraram que a taxa de conversão foi de $29 \%$ durante os primeiros 6 anos de experiência caindo, posteriormente, para $10 \%$. Confirmando, assim, que conforme a equipe adquire maior experiência os resultados melhoram. Não houve recidiva dos sintomas ou de fístula nessa casuística. ${ }^{18}$

Em outro relato com 18 pacientes com diverticulite e fístula ( 15 colovesicais e três colovaginais) todos operados pela via laparoscópica, houve apenas uma conversão para cirurgia aberta e uma recorrência da fístula $(5,5 \%) .{ }^{19}$

Zapletal et al ${ }^{43}$ dividiram 58 pacientes submetidos a LAP eletiva por doença diverticular em dois grupos de acordo com a indicação cirúrgica: diverticulite recidivante $(\mathrm{DR}, \mathrm{n}=32)$ ou diverticulite complicada (DC, n=26). Incluídos no grupo com doença complicada estavam 9 pacientes com fístula. Comparando os dois grupos, não houve diferença na taxa de conversão, mas o tempo cirúrgico foi maior no grupo DC (205 vs $147 \mathrm{~min}$ ). A permanência hospitalar nos casos convertidos também foi, como esperado, maior. Comparando especificamente o subgrupo com fístula com os demais pacientes do grupo com doença complicada, não houve diferença estatisticamente significativa quanto ao tempo operatório. Os autores não observaram recidiva das fístulas nesta casuística e concluíram que a sigmoidectomia laparoscópica é segura também em pacientes com fístula, porém recomendam sua restrição a centros de referência em LAP. Eles também sugerem conversão precoce, assim que houver sinais de dificuldade cirúrgica, visando limitar a morbidade. ${ }^{43}$

\section{Cirurgia eletiva precoce ou tardia?}

As evidências atuais sustentam que a cirurgia eletiva realizada tardiamente ao episódio de diverticulite aguda obtém menores taxas de conversão, morbimortalidade e menor tempo de internação. ${ }^{44,45,46}$

Reissfeld et al ${ }^{44}$ compararam o tratamento da diverticulite aguda por sigmoidectomia laparoscópica eletiva precoce (cinco a oito dias após início do tratamento antibiótico) e tardia (quatro a seis semanas após hospitalização inicial). Foram incluídos 210 pacientes (116 no grupo precoce e 94 no grupo tardio), pareados por idade, escore A.S.A., comorbidades e extensão do processo inflamatório. Ocorreram 10 conversões, sendo 9 no grupo operado precocemente. Apesar do tempo operatório ter sido similar, o grupo precoce apre- 
sentou níveis mais elevados de morbidade $(13,8 \%$ vs $4,2 \%$ ). Os autores concluíram que a cirurgia no intervalo livre de inflamação reduz significativamente a morbidade pós-operatória.

Outro estudo comparando sigmoidectomia laparoscópica precoce e tardia na diverticulite aguda incluiu 178 pacientes e observou maior taxa de conversão no grupo precoce $(37,7 \%$ vs $12,9 \%)$. Os pacientes do grupo precoce também permaneceram mais tempo internados (13,5 vs 10,5 dias). A avaliação histológica da peça revelou inflamação em 75,3\% dos pacientes do grupo precoce e em $23,8 \%$ do grupo tardio. Não houve diferença significativa em relação à morbidade geral e aos custos do tratamento entre os dois grupos. ${ }^{45}$

Chouillard et al ${ }^{46}$ compararam 39 pacientes submetidos a sigmoidectomia laparoscópica até 90 dias da fase aguda da diverticulite e 38 pacientes submetidos ao mesmo procedimento após 90 dias da fase aguda. Novamente, a taxa de conversão, a morbidade e o tempo de internação foram significativamente maiores no grupo precoce ( $13 \%$ vs $5 \% ; 21 \%$ vs $5 \%$ e 7,7 vs 5 dias, respectivamente).

\section{Conduta cirúrgica}

Independentemente da realização da sigmoidectomia de forma eletiva ou na urgência, aber- ta ou laparoscópica, esta deve ser realizada até o reto alto (grau de recomendação B).

A área do cólon descendente/transverso a ser anastomosada não deve estar inflamada, espessada ou hipertrófica e a anastomose deve ser colorretal. ${ }^{25,47}$

\section{CONSIDERAÇÕES FINAIS}

A TC é o melhor exame para avaliar e graduar a severidade da diverticulite aguda. Sinais de diverticulite severa na TC se correlacionam com mau prognóstico precoce e tardio. Diverticulites Hinchey I devem ser tratadas clinicamente. Diverticulites Hinchey II devem, se possível, ser tratadas com antibioticoterapia associada a drenagem percutânea e posterior sigmoidectomia. Diverticulites Hinchey III/ IV devem ser operadas, realizando-se preferencialmente a sigmoidectomia no primeiro tempo.

A cirurgia eletiva é mandatória após diverticulite complicada ou que evoluiu com fístula ou estenose. Sua indicaçãoé razoável nos pacientes que evoluem com sintomas persistentes não responsivos à dieta rica em fibras $\mathrm{e}$ deve ser individualizada nos pacientes que obtiveram sucesso com tratamento clínico inicial e estão assintomáticos.

$\mathrm{O}$ acesso laparoscópico pode ser utilizado por uma equipe treinada tanto em casos de urgência como eletivos.

ABSTRACT: During the past decades the incidence of diverticular disease of the colon and its complications has increased as well as the therapeutic options have expanded. However, there are still remaining doubts about the timing of surgery indication, the access (open vs laparoscopic) and the best approach to complicated diverticulitis. The current decisions are mostly based in old data of poor statistic strength. This review shows the present-day evidence about the treatment of diverticular disease.

Key words: Diverticular Disease of the Colon; Acute Diverticulitis; Treatment.

\section{REFERÊNCIAS}

1- Etzioni DA, Mack TM, Beart RW Jr, Kaiser AM. Diverticulitis in the United States: 1998-2005: changing patterns of disease and treatment. Ann Surg. 2009 Feb;249(2):210-7.

2- Parks TG. Natural history of diverticular disease of the colon. A review of 521 cases. Br Med J. 1969 Dec 13;4(5684):63942.

3- Kang JY, Melville D, Maxwell JD. Epidemiology and management of diverticular disease of the colon. Drugs Aging. 2004;21(4):211-28.
4- Schoetz DJ Jr. Diverticular disease of the colon: a century-old problem. Dis Colon Rectum. 1999 Jun;42(6):703-9.

5- Stollman N, Raskin JB. Diverticular disease of the colon. Lancet. 2004 Feb 21;363(9409):631-9.

6- Rafferty J, Shellito P, Hyman NH, Buie WD; Standards Committee of American Society of Colon and Rectal Surgeons. Practice parameters for sigmoid diverticulitis. Dis Colon Rectum. 2006 Jul;49(7):939-44.

7- Doringer E. Computerized tomography of colonic diverticulitis. Crit Rev Diagn Imaging. 1992;33(5):421-35.

8- Ambrosetti P, Grossholz M, Becker C, Terrier F, Morel P. Computed tomography in acute left colonic diverticulitis. $\mathrm{Br}$ J Surg. 1997 Apr;84(4):532-4. 
9- Larson DM, Masters SS, Spiro HM.Medical and surgical therapy in diverticular disease: a comparative study. Gastroenterology. 1976 Nov;71(5):734-7.

10- Detry R, Jamez J, Kartheuser A, Zech F, Vanheuverzwijn R, Hoang P, Kestens PJ.Acute localized diverticulitis: optimum management requires accurate staging. Int J Colorectal Dis. 1992 Feb;7(1):38-42.

11- Ambrosetti P, Chautems R, Soravia C, Peiris-Waser N, Terrier F. Long-term outcome of mesocolic and pelvic diverticular abscesses of the left colon: a prospective study of 73 cases. Dis Colon Rectum. 2005 Apr;48(4):787-91.

12- Nagorney DM, Adson MA, Pemberton JH. Sigmoid diverticulitis with perforation and generalized peritonitis. Dis Colon Rectum 1985;28:71-5.

13- Belmonte C, Klas JV, Perez JJ, Wong WD, Rothenberger DA, Goldberg SM, et al. The hartmann procedure. First choice or last resort in diverticular disease. Arch Surg 1996;131:612-7.

14- Bretagnol F, Pautrat K, Mor C, Benchellal Z, Huten N, Calan L. Emergency Laparoscopic Management of Perforated Sigmoid Diverticulitis: A Promising Alternative to More Radical Procedures. J Am Coll Surg 2008;206:654-657

15- Franklin ME Jr, Portillo G, Treviño JM, Gonzalez JJ, Glass JL. Long-term experience with the laparoscopic approach to perforated diverticulitis plus generalized peritonitis. World $\mathrm{J}$ Surg. 2008 Jul;32(7):1507-11.

16- Myers E, Hurley M, O’Sullivan GC, Kavanagh D, Wilson I, Winter DC. Laparoscopic peritoneal lavage for generalized peritonitis due to perforated diverticulitis. British Journal of Surgery 2008; 95: 97-101

17- Taylor CJ, Layani L, Ghusn MA, White SI. Perforated diverticulitis managed by laparoscopic lavage. ANZ J. Surg. 2006; 76: 962-965

18- Engledow AH, Pakzad F, Ward NJ, Arulampalam T, Motson RW. Laparoscopic resection of diverticular fistulae: a 10-year experience. Colorectal Dis. 2007 Sep;9(7):632-4.

19- Menenakos E, Hahnloser D, Nassiopoulos K, Chanson C, Sinclair V, Petropoulos P. Laparoscopic surgery for fistulas that complicate diverticular disease. Langenbecks Arch Surg.2003 Jul;388(3):189-93.

20- Scheidbach H, Schneider C, Rose J, Konradt J, Gross E, Bärlehner E, Pross M, Schmidt U, Köckerling F, Lippert H. Laparoscopic approach to treatment of sigmoid diverticulitis: changes in the spectrum of indications and results of a prospective, multicenter study on 1.545 patients. Dis Colon Rectum. 2004 Nov;47(11):1883-8.

21- Weber WP, Guller U, Jain NB, Pietrobon R, Oertli D. Impact of surgeon and hospital caseload on the likelihood of performing laparoscopic vs open sigmoid resection for diverticular disease: a study based on 55,949 patients. Arch Surg. 2007 Mar;142(3):253-9.

22- Hassan I, Cima RR, Larson DW, Dozois EJ, Byrne MMO, Larson DR, Pemberton JH. The Impact of Uncomplicated and Complicated Diverticulitis on Laparoscopic Surgery
Conversion Rates and Patient Outcomes. Surg Endosc (2007) 21: 1690-1694

23- Köhler L, Sauerland S, Neugebauer E. Diagnosis and treatment of diverticular disease: results of a consensus development conference. The Scientific Committee of the European Association for Endoscopic Surgery. Surg Endosc. 1999 Apr;13(4):430-6.

24- Kaiser AM, Jiang JK, Lake JP, Ault G, Artinyan A, GonzalezRuiz C, Essani R, Beart RW Jr. The management of complicated diverticulitis and the role of computed tomography. Am J Gastroenterol. 2005 Apr;100(4):910-7.

25- Wong WD, Wexner SD, Lowry A, Vernava A 3rd, Burnstein M, Denstman F, Fazio V, Kerner B, Moore R, Oliver G, Peters W, Ross T, Senatore P, Simmang C. Practice parameters for the treatment of sigmoid diverticulitis-supporting documentation. The Standards Task Force. The American Society of Colon and Rectal Surgeons. Dis Colon Rectum. 2000 Mar;43(3):290-7.

26- Stollman NH, Raskin JB.Diagnosis and management of diverticular disease of the colon in adults. Ad Hoc Practice Parameters Committee of the American College of Gastroenterology. Am J Gastroenterol. 1999 Nov;94(11):311021.

27- Peppas G, Bliziotis IA, Oikonomaki D, Falagas ME.Outcomes after medical and surgical treatment of diverticulitis: a systematic review of the available evidence. J Gastroenterol Hepatol. 2007 Sep;22(9):1360-8.

28- Anaya DA, Flum DR. Risk of emergency colectomy and colostomy in patients with diverticular disease. Arch Surg. $2005 \mathrm{Jul} ; 140(7): 681-5$.

29- Broderick-Villa G, Burchette RJ, Collins JC, Abbas MA, Haigh PI Hospitalization for acute diverticulitis does not mandate routine elective colectomy. Arch Surg. 2005 Jun;140(6):57681.

30- Chapman J, Davies M, Wolff B, Dozois E, Tessier D, Harrington J, Larson D.Complicated diverticulitis: is it time to rethink the rules? Ann Surg. 2005 Oct;242(4):57681.

31- Salem TA, Molloy RG, O’Dwyer PJ. Prospective, five-year follow-up study of patients with symptomatic uncomplicated diverticular disease. Dis Colon Rectum. 2007 Sep;50(9):14604.

32- Chautems RC, Ambrosetti P, Ludwig A, Mermillod B, Morel P, Soravia C.Long-term follow-up after first acute episode of sigmoid diverticulitis: is surgery mandatory?: a prospective study of 118 patients. Dis Colon Rectum. 2002 Jul;45(7):9626.

33- Salem L, Veenstra DL, Sullivan SD, Flum DR.The timing of elective colectomy in diverticulitis: a decision analysis. J Am Coll Surg. 2004 Dec;199(6):904-12.

34- Richards RJ, Hammitt JK. Timing of prophylactic surgery in prevention of diverticulitis recurrence: a cost-effectiveness analysis. Dig Dis Sci. 2002 Sep;47(9):1903-8. 
35- Pautrat K, Bretagnol F, Huten N, de Calan L. Acute diverticulitis in very young patients: a frequent surgical management. Dis Colon Rectum. 2007 Apr;50(4):472-7.

36- Vignati PV, Welch JP, Cohen JL. Long-term management of diverticulitis in young patients. Dis Colon Rectum. 1995 Jun;38(6):627-9.

37- Jones OM, Stevenson AR, Clark D, Stitz RW, Lumley JW. Laparoscopic Resection for Diverticular Disease - Follow-up of 500 Consecutive Patients. Ann Surg.2008 Dec;248(6):10927.

38- Schwandner O, Farke S, Bruch HP. Laparoscopic colectomy for diverticulitis is not associated with increased morbidity when compared with non-diverticular disease. Int J Colorectal Dis. 2005 Mar;20(2):165-72.

39- Klarenbeek BR, Veenhof AA, Bergamaschi R, van der Peet DL, van den Broek WT, de Lange ES, Bemelman WA, Heres P, Lacy AM, Engel AF, Cuesta MA. Laparoscopic sigmoid resection for diverticulitis decreases major morbidity rates: a randomized control trial: short-term results of the Sigma Trial. Ann Surg. 2009 Jan;249(1):39-44.

40- Shapiro SB, Lambert PJ, Mathiason MA. A comparison of open and laparoscopic techniques in elective resection for diverticular disease. WMJ. 2008 Sep;107(6):287-91.

41- Gonzalez R, Smith CD, Mattar SG, Venkatesh KR, Mason E, Duncan T, Wilson R, Miller J, Ramshaw BJ. Laparoscopic vs open resection for the treatment of diverticular disease. Surg Endosc. 2004 Feb;18(2):276-80.

42- Senagore AJ, Duepree HJ, Delaney CP, Dissanaike S, Brady KM, Fazio VW. Cost structure of laparoscopic and open sigmoid colectomy for diverticular disease: similarities and differences. Dis Colon Rectum. 2002 Apr;45(4):485-90.
43- Zapletal C, Woeste G, Bechstein WO, Wullstein C. Laparoscopic sigmoid resections for diverticulitis complicated by abscesses or fistulas. Int J Colorectal Dis (2007) 22:15151521

44- Reissfelder C, Buhr HJ, Ritz JP. What is the optimal time of surgical intervention after an acute attack of sigmoid diverticulitis: early or late elective laparoscopic resection? Dis Colon Rectum. 2006 Dec;49(12):1842-8.

45- Zingg U, Pasternak I, Guertler L, Dietrich M, Wohlwend KA, Metzger U. Early vs. delayed elective laparoscopic-assisted colectomy in sigmoid diverticulitis: timing of surgery in relation to the acute attack. Dis Colon Rectum. 2007 Nov;50(11):19117.

46- Chouillard E, Benhaim L, Ata T, Etienne JC, Ghiles E, Fingerhut A.Elective laparoscopic colectomy in uncomplicated diverticulitis: when should surgery be performed Cir Esp. 2007 Apr;81(4):207-12.

47- Thaler K, Baig MK, Berho M, Weiss EG, Nogueras JJ, Arnaud JP, Wexner SD, Bergamaschi R. Determinants of recurrence after sigmoid resection for uncomplicated diverticulitis. Dis Colon Rectum. 2003 Mar;46(3):385-8.

\section{Endereço para correspondência:} ANDRÉ RONCON DIAS

Al. Ministro Rocha Azevedo, 644, apto 11.

Cerqueira César, São Paulo - SP, Brasil.

CEP:01410-000

E-mail: roncon86@hotmail.com 